\title{
Synthesis, Crystal structure of hepta (pyridinium) bis (hexachlorobismuthate (III)) nitrate $\left[\mathrm{C}_{5} \mathrm{H}_{6} \mathrm{~N}\right]_{7}\left(\mathrm{BiCl}_{6}\right)_{2}\left(\mathrm{NO}_{3}\right)$
}

Fayçal Ben Tahar ${ }^{a^{*}}$, Perez Olivier $^{\mathrm{b}}$, Slaheddine Chaabouni ${ }^{\mathrm{a}}$

a'Laboratoire des Sciences de Matériaux et de l'Environnement (MESLab), Faculté des Sciences de Sfax (FSS), Université de Sfax, Route de Soukra - km 3,5 - BP 1171, 3018- Sfax, Tunisia.

E-mail adress : f_bentahar@yahoo.fr; chaabouni_slaheddine@yahoo.fr bLaboratoire CRISMAT, UMR 6508 CNRS, ENSICAEN- 6 Boulevard du Marechal Juin- 14050- Caen- Cedex 4- France.

\section{E-mail adress : olivier.perez@ensicaen.fr}

\section{Abstract}

An hepta (pyridinium) bis (hexachlorobismuthate (III)) nitrate, $\left(\mathrm{C}_{5} \mathrm{H}_{6} \mathrm{~N}\right)_{7}\left(\mathrm{BiCl}_{6}\right)_{2}\left(\mathrm{NO}_{3}\right)$ crystallizes at room temperature in the monoclinic system, space group $\mathrm{P} 2{ }_{1} / \mathrm{n}$, with the following unit-cell parameters: $a=9.555(1) \AA$, $b=16.847(1) \AA$, $c=32.522(1) \AA, \beta=94.37^{\circ}, V=5219.8 \AA^{3}$ and four molecules per unit cell. Its crystal structure was determined and refined down to $R_{1}=0.0504, w R_{2}=0.0667$. The structure of the title compound, $\left(\mathrm{C}_{5} \mathrm{H}_{6} \mathrm{~N}\right)_{7}\left(\mathrm{BiCl}_{6}\right)_{2}\left(\mathrm{NO}_{3}\right)$ consists of seven monoprotonated pyridinium $\left(\mathrm{C}_{5} \mathrm{H}_{6} \mathrm{~N}\right)^{+}$cations, two independent octahedron $\left[\mathrm{BiCl}_{6}\right]^{3-}$ and an isolated $\mathrm{NO}_{3}^{-}$anion. These entities are linked together through $\mathrm{N}-\mathrm{H}^{\cdots \cdots} \cdot \mathrm{Cl}$ and $\mathrm{N}-\mathrm{H}^{\cdots \cdot} \mathrm{O}$ hydrogen bonds, originating from the $\left(\mathrm{C}_{5} \mathrm{H}_{6} \mathrm{~N}\right)^{+}$groups and the isolated anion of nitrate to forming a three dimensional network.

\section{Indexing terms/Keywords}

Organic-inorganic hybrid material; Crystal structure; Halogenobismuthates(III)

* Corresponding author: Dr. Ben Tahar Fayçal

Tel: 21698949214 , Fax: 21674274437

E-mail address: $\mathbf{f}$ bentahar@yahoo.fr

Address: Soukra Road - km 3.5 - B.P. 1171, 3018 Sfax - Tunisia

\section{Council for Innovative Research}

Peer Review Research Publishing System

Journal: Journal of Advances in Chemistry

Vol. 9, No. 2

editorjaconline@gmail.com

www.cirworld.org/journals 


\section{Introduction}

Halogenoantimonates(III) and halogenobismuthates(III) containing various organic cations form a large class of the organic- inorganic hybrid materials [1-5]. These molecular-ionic crystals are built up of the inorganic anions and organic cations. The compounds form various anionic structures in the solid state. The anionic sublattice is built up of either the simple discrete octahedra or one-dimensional chains or two- or three-dimensional anionic framework. In the majority of these hybrid materials the anionic moieties are rigid, whereas the organic cations, placed in large cavities of the anionic substructure, exhibit usually reorientational disorder.

The halogenoantimonates (III) and halogenobismuthates (III) evoke much interest because their single crystals exhibit the polar, non-linear or ferroic properties. The change in the dynamical state of cations is responsible for the mechanisms of the numerous structural phase transitions found in these materials. Such a mechanism of structural phase transitions was classified as 'order-disorder'. A long range ferroelectric order of the dipoles has been found in the compounds containing generally the small in size ammonium cations (like monomethyl-, dimethyl- or trimethyl-ammonium) [6-10].

A novel group of crystals, containing heteroaromatic cations like: pyridinium, substituted pyridinium and imidazolium ones, have been recently synthesized and characterized [11-13]. Since aromatic heterocyclic cations are bestowed a significant electric dipole moment thus some halogenoantimonates (III) and halogenobismuthates (III) containing these cations form strongly polar structures. We have studied three pyridinium halogenobismuthates (III), i.e. $\left(\mathrm{C}_{5} \mathrm{H}_{5} \mathrm{NH}_{6} \mathrm{Bi}_{4} \mathrm{Cl}_{18}[14]\right.$, $\left(\mathrm{C}_{5} \mathrm{H}_{5} \mathrm{NH}\right) \mathrm{BiCl}_{4}$ [15] and $\left(\mathrm{C}_{5} \mathrm{H}_{5} \mathrm{NH}\right)_{5} \mathrm{Bi}_{2} \mathrm{Br}_{11}$ [16]. The ferroelectricity was found, however, only in the compound of the $\mathrm{R}_{5} \mathrm{Bi}_{2} \mathrm{X}_{11}$ composition, $\left(\mathrm{C}_{5} \mathrm{H}_{5} \mathrm{NH}\right)_{5} \mathrm{Bi}_{2} \mathrm{Br}_{11}$. This latter compound is a ferroelectric below the Curie temperature of $118 \mathrm{~K}$. Among a dozen of the substituted pyridinium compounds, studied so far, a ferroelectricity is confirmed merely in a case of 4-aminopyridinium tetrachloroantimonate (III), $\left(4-\mathrm{NH}_{2} \mathrm{C}_{5} \mathrm{H}_{4} \mathrm{NH}\right) \mathrm{SbCl}_{4}$ [17], below $\mathrm{T}_{\mathrm{c}}=270.5 \mathrm{~K}$. The presence of polar properties in the materials described above is usually a result of the organic cations dynamics, which play a key role in the induction of ferroelectricity. The results, obtained by us for the group of ionic compounds containing aromatic cations, trace a direction of search for the novel polar materials.

Herein we report the synthesis and the crystal structural characterization by X-ray diffraction of the hepta (pyridinium) bis (hexachlorobismuthate (III)) nitrate.

\section{Experimental section}

\section{II.1.Synthesis}

Transparents and colorless crystals of $\left[\mathrm{C}_{5} \mathrm{H}_{5} \mathrm{NH}\right]_{7}\left(\mathrm{BiCl}_{6}\right)_{2}\left(\mathrm{NO}_{3}\right)$ were synthesized by the addition of pyridinium to a solution of bismuth (III) nitrate (molar ratio 7/2) in concentrated hydrochloric acid (36\%). Single crystals were grown by the slow evaporation of an aqueous solution at room temperature.

The chemical reaction of the title compound was the following :

$$
7 \mathrm{C}_{5} \mathrm{H}_{5} \mathrm{~N}+2 \mathrm{Bi}\left(\mathrm{NO}_{3}\right)_{3}+12 \mathrm{HCl} \stackrel{\mathrm{H}_{2} \mathrm{O} / \mathrm{H}}{\longrightarrow}\left[\mathrm{C}_{5} \mathrm{H}_{6} \mathrm{~N}\right]_{7} \mathrm{Cl}\left(\mathrm{BiCl}_{6}\right)_{2}\left(\mathrm{NO}_{3}\right)+5 \mathrm{HNO}_{3}
$$

\section{II.2.Single crystal Structure determination}

X-ray data collection was carried out on a Bruker Kappa Apex II CCD diffractometer with graphite monochromated Mo $\mathrm{K}_{\mathrm{o}}$ radiation. Bismuth and chlorine atoms positions were located using the direct method with program SHELXS-97 [18] program, while the carbon, oxygen, and nitrogen atoms were found from successive difference Fourier calculations using SHELXL97 [19] computer program. The structure is refined by full matrix least-squares using anisotropic temperature factors for all non-hydrogen atoms. All hydrogen atoms were geometrically fixed at the calculated positions attached to their parent atoms and treated as riding atoms. Crystal data, collected reflections, and parameters of the final refinement are reported in Table 1.

Table 1: Experimental data for X-ray diffraction study of $\left[\mathrm{C}_{5} \mathrm{H}_{5} \mathrm{NH}\right]_{7}\left(\mathrm{BiCl}_{6}\right)_{2}\left(\mathrm{NO}_{3}\right)$

\begin{tabular}{ll}
\hline Crystal data & \\
Empirical formula & $\mathrm{C}_{35} \mathrm{H}_{42} \mathrm{Bi}_{2} \mathrm{Cl}_{12} \mathrm{~N}_{8} \mathrm{O}_{3}$ \\
Formula weight $(\mathrm{g} / \mathrm{mol})$ & 1466,13 \\
Crystal system, Space group & monoclinic, $P 2{ }_{1} / \mathrm{n}$ \\
$a(\AA)$ & $9.555(1)$ \\
$b(\AA)$ & $16.847(1)$ \\
$c(\AA)$ & $32.522(1)$ \\
$\beta$ & $94.37(1)^{\circ}$ \\
$V\left(\AA^{3}\right)$ & $5219.8(0)$ \\
$Z$ & 4 \\
\hline
\end{tabular}




\begin{tabular}{ll}
\hline$D_{\text {calc }}\left(\mathrm{g} \mathrm{cm}^{-3}\right)$ & 1.866 \\
$\mu\left(\mathrm{mm}^{-1}\right)$ & 7.389 \\
$F(000)$ & 2808
\end{tabular}

Crystal size (mm)

$0.25 \times 0.20 \times 0.15$

Crystal habit

Colorless

Data collection

Diffractometer

Monochromator

BrukerKappaApex II CCD diffractometer Graphite

Radiation type, $\lambda(\AA)$

Mo-K/ a, 0.71073

$T(\mathrm{~K})$

293(2)

$\Theta$ Range $\left({ }^{\circ}\right)$

$1.26-34.99$

Indexes range

$-11 \leq h \leq 15$

$-26 \leq k \leq 27$

$-52 \leq I \leq 51$

Absorption correction

$T_{\min } / T_{\max }$

numerical

$0.2595 / 0.4036$

84321

21099

Measured reflections

12702

0.0354

Observed refl. $(I>2 \sigma())$

$R_{\text {int }}$

Refinement

Refinement on

$F^{2}$

21099/5/541

Data/restraints/parameters

$R 1=0.0504$

$\left.R\left(F_{0}^{2}\right)>2 \sigma\left(F_{0}^{2}\right)\right]$

$w R 2=0.0667$

1.021

GooF $=S$

926364

CCDC deposition number

\section{Results and discussion}

The final atomic coordinates obtained from the single crystal refinement with $\bigcup_{\text {eq }}$ are given in Table 2. The anisotropic displacement parameters are shown in Table 3. Interatomic distances and bond angles schemes are listed in Table 4.

The asymmetric unit of our component, $\left[\mathrm{C}_{5} \mathrm{H}_{5} \mathrm{NH}\right]_{7}\left(\mathrm{BiCl}_{6}\right)_{2}\left(\mathrm{NO}_{3}\right)$, consists of two distinct octahedra $\left[\mathrm{BiCl}_{6}\right]^{3-}$, isolated $\mathrm{NO}_{3}$ anion and seven monoprotonated pyridinium cations as shown in Fig. 1. 


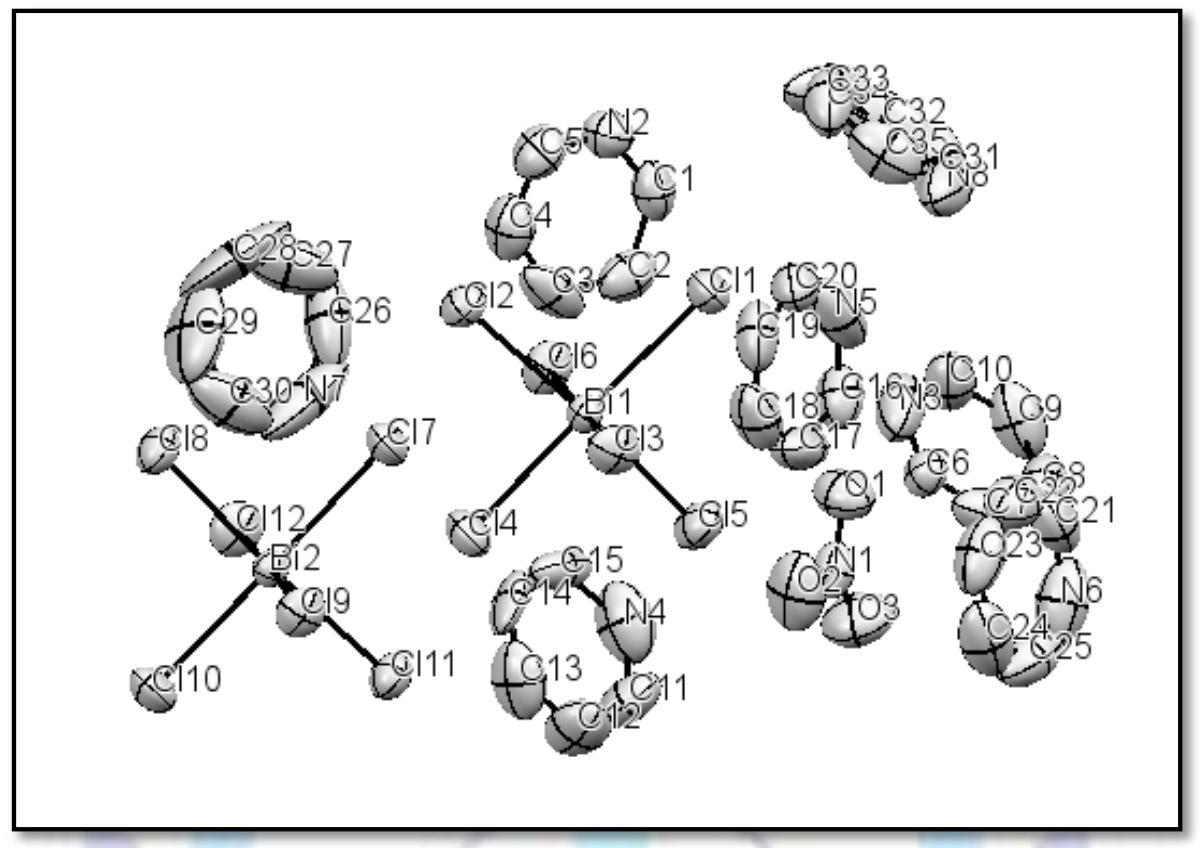

Figure 1 : The asymmetric unit of $\left[\mathrm{C}_{5} \mathrm{H}_{5} \mathrm{NH}\right]_{7}\left(\mathrm{BiCl}_{6}\right)_{2}\left(\mathrm{NO}_{3}\right)$, showing the atom-numbering scheme

Table 2: Atomic coordinates and $U_{\text {eq }}$ or $U_{\text {iso }}$ for $\left[\mathrm{C}_{5} \mathrm{H}_{5} \mathrm{NH}\right]_{7}\left(\mathrm{BiCl}_{6}\right)_{2}\left(\mathrm{NO}_{3}\right)$

\begin{tabular}{|c|c|c|c|c|}
\hline Atomes & $\mathbf{x}$ & $\mathbf{y}$ & $\mathbf{z}$ & Uéq \\
\hline $\mathrm{Bi} 1$ & $0.044479(13)$ & $0.648857(9)$ & $0.285559(4)$ & $0.03835(4)$ \\
\hline $\mathrm{Bi} 2$ & $0.758222(13)$ & $0.740706(8)$ & $0.491828(4)$ & $0.03762(4)$ \\
\hline $\mathrm{Cl} 1$ & $0.21692(11)$ & $0.72253(8)$ & $0.23300(4)$ & $0.0634(3)$ \\
\hline $\mathrm{Cl} 2$ & $0.26306(10)$ & $0.57334(7)$ & $0.32870(4)$ & $0.0562(3)$ \\
\hline $\mathrm{Cl} 3$ & $0.03392(12)$ & $0.51559(8)$ & $0.23403(4)$ & $0.0674(3)$ \\
\hline $\mathrm{Cl} 4$ & $-0.12433(11)$ & $0.57297(8)$ & $0.33372(4)$ & $0.0654(3)$ \\
\hline $\mathrm{Cl} 5$ & $-0.18081(11)$ & $0.71957(8)$ & $0.24617(4)$ & $0.0687(3)$ \\
\hline $\mathrm{Cl} 6$ & $0.07738(12)$ & $0.76756(7)$ & $0.33769(4)$ & $0.0638(3)$ \\
\hline $\mathrm{Cl} 7$ & $0.93579(11)$ & $0,81443(8)$ & $0,44270(4)$ & $0,0613(3)$ \\
\hline $\mathrm{Cl} 8$ & $-0.99095(11)$ & $0.67426(8)$ & $0.53184(4)$ & $0.0628(3)$ \\
\hline $\mathrm{Cl9}$ & $0.73347(11)$ & $0.61258(7)$ & $0.44107(4)$ & $0.0600(3)$ \\
\hline Cl10 & $0.58374(12)$ & $0.67365(8)$ & $0.54308(4)$ & $0.0713(3)$ \\
\hline Cl11 & $0.54242(10)$ & $0.80979(7)$ & $0.44775(3)$ & $0.0576(3)$ \\
\hline $\mathrm{Cl} 12$ & $0.77755(13)$ & $0.86515(8)$ & $0.54412(4)$ & $0.0687(3)$ \\
\hline $\mathrm{O} 1$ & $0.9038(4)$ & $0.6237(2)$ & $0.13693(14)$ & $0.0905(12)$ \\
\hline $\mathrm{O} 2$ & $0.7792(5)$ & $0.5196(3)$ & $0.13845(15)$ & $0.1150(16)$ \\
\hline $\mathrm{O3}$ & $0.6832(4)$ & $0.6313(3)$ & $0.13652(16)$ & $0.1136(16)$ \\
\hline N1 & $0.7854(5)$ & $0.5915(3)$ & $0.13727(12)$ & $0.0613(10)$ \\
\hline N2 & $0.1329(4)$ & $0.5251(3)$ & $0.13220(14)$ & $0.0719(12)$ \\
\hline N3 & $0.9661(6)$ & $0.8279(5)$ & $0.15846(17)$ & $0.124(2)$ \\
\hline N4 & $-0.1099(8)$ & $0.3541(3)$ & $0.1720(2)$ & $0.120(2)$ \\
\hline N5 & $0.4614(9)$ & $0.5583(4)$ & $0.24511(18)$ & $0.127(2)$ \\
\hline N6 & $0.6850(9)$ & $0.6524(5)$ & $0.02821(18)$ & $0.120(2)$ \\
\hline
\end{tabular}




\begin{tabular}{|c|c|c|c|c|}
\hline N7 & $0.0986(12)$ & $0.6144(10)$ & $0.4288(3)$ & $0.172(6)$ \\
\hline N8 & $0.3469(6)$ & $0.8201(7)$ & $0.12434(18)$ & $0.138(3)$ \\
\hline C1 & $0.2495(6)$ & $0.5616(3)$ & $0.14554(16)$ & $0.0712(14)$ \\
\hline C2 & $0.3759(5)$ & $0.5293(4)$ & $0.13689(17)$ & $0.0733(15)$ \\
\hline C3 & $0.3771(6)$ & $0.4615(4)$ & $0.11502(19)$ & $0.0830(18)$ \\
\hline C4 & $0.2538(8)$ & $0.4267(4)$ & $0.10216(19)$ & $0.0875(18)$ \\
\hline C5 & $0.1343(6)$ & $0.4592(4)$ & $0.11105(19)$ & $0.0799(16)$ \\
\hline C6 & $0.8686(5)$ & $0.7878(3)$ & $0.13562(15)$ & $0.0535(11)$ \\
\hline $\mathrm{C} 7$ & $0.7845(6)$ & $0.8221(4)$ & $0.10967(19)$ & $0.0846(18)$ \\
\hline C8 & $0.7904(7)$ & $0.9002(5)$ & $0.1028(2)$ & $0.093(2)$ \\
\hline C9 & $0.8847(8)$ & $0.9434(4)$ & $0.1248(2)$ & $0.0944(19)$ \\
\hline C10 & $0.9742(7)$ & $0.9101(4)$ & $0.1522(2)$ & $0.0860(18)$ \\
\hline C11 & $0.0079(6)$ & $0.3152(5)$ & $0.1838(2)$ & $0.0905(19)$ \\
\hline C12 & $0.0230(7)$ & $0.2424(4)$ & $0.1745(2)$ & $0.100(2)$ \\
\hline C13 & $-0.0809(11)$ & $0.2038(4)$ & $0.1528(2)$ & $0.109(2)$ \\
\hline C14 & $-0.1941(7)$ & $0.2402(5)$ & $0.14071(17)$ & $0.0782(18)$ \\
\hline C15 & $-0.2143(5)$ & $0.3122(6)$ & $0.14944(19)$ & $0.093(2)$ \\
\hline C16 & $0.6059(7)$ & $0.5551(4)$ & $0.24427(18)$ & $0.0833(17)$ \\
\hline C17 & $0.6649(6)$ & $0.4874(6)$ & $0.2394(2)$ & $0.099(2)$ \\
\hline C18 & $0.5848(9)$ & $0.4206(4)$ & $0.2348(2)$ & $0.101(2)$ \\
\hline C19 & $0.4452(8)$ & $0.4273(5)$ & $0.23570(18)$ & $0.095(2)$ \\
\hline C20 & $0.3864(5)$ & $0.4934(5)$ & $0.24094(17)$ & $0.0741(16)$ \\
\hline $\mathrm{C} 21$ & $0.8287(10)$ & $0.6546(5)$ & $0.0283(2)$ & $0.099(2)$ \\
\hline C22 & $0.8972(6)$ & $0.5873(7)$ & $0.0326(2)$ & $0.105(3)$ \\
\hline C23 & $0.8251(10)$ & $0.5196(5)$ & $0.0359(2)$ & $0.104(2)$ \\
\hline C24 & $0.6870(11)$ & $0.5219(5)$ & $0.0358(2)$ & $0.107(2)$ \\
\hline C25 & $0.6207(7)$ & $0.5877(7)$ & $0.0319(2)$ & $0.101(2)$ \\
\hline C26 & $0.213(2)$ & $0.6586(5)$ & $0.4292(3)$ & $0.149(5)$ \\
\hline C27 & $0.3274(12)$ & $0.6302(9)$ & $0.4465(4)$ & $0.148(4)$ \\
\hline C28 & $0.3240(13)$ & $0.5630(10)$ & $0.4632(4)$ & $0.175(6)$ \\
\hline C29 & $0.214(2)$ & $0.5197(5)$ & $0.4640(4)$ & $0.147(4)$ \\
\hline C30 & $0.1049(12)$ & $0.5468(10)$ & $0.4470(5)$ & $0.151(5)$ \\
\hline C31 & $0.3605(13)$ & $0.8918(7)$ & $0.1470(4)$ & $0.141(5)$ \\
\hline C32 & $0.4364(14)$ & $0.8937(5)$ & $0.1794(4)$ & $0.134(4)$ \\
\hline C33 & $0.5117(8)$ & $0.8355(9)$ & $0.1913(3)$ & $0.132(4)$ \\
\hline C34 & $0.5117(8)$ & $0.7712(6)$ & $0.1724(3)$ & $0.109(3)$ \\
\hline C35 & $0.4306(9)$ & $0.7609(4)$ & $0.1390(3)$ & $0.100(2)$ \\
\hline $\mathrm{H} 1$ & 0.2464 & 0.6085 & 0.1606 & 0.085 \\
\hline $\mathrm{H} 2$ & 0.0535 & 0.5452 & 0.1376 & 0.086 \\
\hline $\mathrm{H} 2 \mathrm{~A}$ & 0.4596 & 0.5539 & 0.1460 & 0.088 \\
\hline $\mathrm{H} 3$ & 1.0221 & 0.8043 & 0.1764 & 0.149 \\
\hline
\end{tabular}




\begin{tabular}{|c|c|c|c|c|}
\hline H311 & 0.4617 & 0.4388 & 0.1088 & 0.100 \\
\hline $\mathrm{H} 4$ & 0.2533 & 0.3799 & 0.0870 & 0.105 \\
\hline $\mathrm{H} 4 \mathrm{~A}$ & -0.1206 & 0.4033 & 0.1780 & 0.143 \\
\hline H5 & 0.0499 & 0.4349 & 0.1022 & 0.096 \\
\hline $\mathrm{H} 5 \mathrm{~A}$ & 0.4207 & 0.6032 & 0.2484 & 0.152 \\
\hline $\mathrm{H} 6$ & 0.8624 & 0.7331 & 0.1389 & 0.064 \\
\hline $\mathrm{H} 6 \mathrm{~A}$ & 0.6381 & 0.6959 & 0.0256 & 0.144 \\
\hline $\mathrm{H} 7$ & 0.7162 & 0.7921 & 0.0948 & 0.102 \\
\hline H7A & 0.0210 & 0.6308 & 0.4165 & 0.206 \\
\hline $\mathrm{H} 8$ & 0.7290 & 0.9239 & 0.0829 & 0.112 \\
\hline $\mathrm{H} 8 \mathrm{~A}$ & 0.2894 & 0.8149 & 0.1028 & 0.165 \\
\hline $\mathrm{H} 9$ & 0.8877 & 0.9980 & 0.1207 & 0.113 \\
\hline $\mathrm{H} 10$ & 1.0415 & 0.9405 & 0.1672 & 0.103 \\
\hline $\mathrm{H} 11$ & 0,0800 & 0.3415 & 0.1991 & 0.109 \\
\hline $\mathrm{H} 12$ & 0.1059 & 0.2160 & 0.1828 & 0.121 \\
\hline $\mathrm{H} 13$ & -0.0705 & 0.1504 & 0.1464 & 0.131 \\
\hline $\mathrm{H} 14$ & -0.2638 & 0.2129 & 0.1250 & 0.094 \\
\hline $\mathrm{H} 15$ & -0.2986 & 0.3369 & 0.1408 & 0.112 \\
\hline $\mathrm{H} 16$ & 0.6596 & 0.6011 & 0.2472 & 0.100 \\
\hline $\mathrm{H} 17$ & 0.7619 & 0.4841 & 0.2390 & 0.119 \\
\hline $\mathrm{H} 18$ & 0.6261 & 0.3714 & 0.2311 & 0.122 \\
\hline $\mathrm{H} 19$ & 0.3900 & 0.3820 & 0.2324 & 0.113 \\
\hline $\mathrm{H} 2 \mathrm{O}$ & 0.2894 & 0.4959 & 0.2418 & 0.089 \\
\hline $\mathrm{H} 21$ & 0.8760 & 0.7022 & 0.0253 & 0.119 \\
\hline $\mathrm{H} 22$ & 0.9947 & 0.5866 & 0.0335 & 0.126 \\
\hline $\mathrm{H} 23$ & 0.8721 & 0.4713 & 0.0382 & 0.124 \\
\hline H24 & 0.6372 & 0.4750 & 0.0387 & 0.128 \\
\hline $\mathrm{H} 25$ & 0.5234 & 0.5879 & 0.0317 & 0.122 \\
\hline H26 & 0.2106 & 0.7087 & 0.4173 & 0.179 \\
\hline H27 & 0.4107 & 0.6588 & 0.4468 & 0.178 \\
\hline H28 & 0.4074 & 0.5433 & 0.4758 & 0.210 \\
\hline H29 & 0.2163 & 0.4703 & 0.4768 & 0.177 \\
\hline $\mathrm{H} 30$ & 0.0232 & 0.5169 & 0.4473 & 0.182 \\
\hline $\mathrm{H} 31$ & 0.3127 & 0.9371 & 0.1376 & 0.169 \\
\hline H32 & 0.4381 & 0.9394 & 0.1955 & 0.161 \\
\hline H33 & 0.5699 & 0.8407 & 0.2154 & 0.158 \\
\hline H34 & 0.5703 & 0.7303 & 0.1822 & 0.131 \\
\hline H35 & 0.4305 & 0.7126 & 0.1251 & 0.120 \\
\hline
\end{tabular}


Table 3: Anisotropic displacement parameters $\left(\AA^{2}\right)$ for $\left[\mathrm{C}_{5} \mathrm{H}_{5} \mathrm{NH}\right]_{7}\left(\mathrm{BiCl}_{6}\right)_{2}\left(\mathrm{NO}_{3}\right)$

\begin{tabular}{|c|c|c|c|c|c|c|}
\hline Atomes & U11 & U22 & U33 & U23 & U13 & U12 \\
\hline Bi1 & $0.03903(6)$ & $0.03560(8)$ & $0.04035(8)$ & $-0.00004(6)$ & $0.00259(5)$ & $0.00134(5)$ \\
\hline $\mathrm{Bi} 2$ & $0.03866(6)$ & $0.03664(8)$ & $0.03720(8)$ & $-0.00024(6)$ & $0.00053(5)$ & $-0.00083(5)$ \\
\hline $\mathrm{Cl} 1$ & $0.0552(5)$ & $0.0708(8)$ & $0.0650(8)$ & $0.0167(6)$ & $0.0102(5)$ & $-0.0056(5)$ \\
\hline $\mathrm{Cl} 2$ & $0.0532(5)$ & $0.0518(7)$ & $0.0620(7)$ & $-0.0001(5)$ & $-0.0065(4)$ & $0.0048(4)$ \\
\hline $\mathrm{Cl} 3$ & $0.0723(7)$ & $0.0695(9)$ & $0.0600(8)$ & $-0.0228(6)$ & $0.0038(5)$ & $-0.0137(6)$ \\
\hline $\mathrm{Cl} 4$ & $0.0614(6)$ & $0.0656(8)$ & $0.0722(8)$ & $0.0154(6)$ & $0.0241(5)$ & $0.0009(5)$ \\
\hline $\mathrm{Cl} 5$ & $0.0591(6)$ & $0.0618(8)$ & $0.0824(9)$ & $0.0115(7)$ & $-0.0123(5)$ & $0.0068(5)$ \\
\hline $\mathrm{Cl} 6$ & $0.0799(7)$ & $0.0497(7)$ & $0.0601(8)$ & $-0.0154(6)$ & $-0.0061(5)$ & $0.0071(5)$ \\
\hline $\mathrm{Cl} 7$ & $0.0565(5)$ & $0.0751(8)$ & $0.0535(7)$ & $0.0127(6)$ & $0.0114(5)$ & $-0.0121(5)$ \\
\hline $\mathrm{Cl} 8$ & $0.0557(5)$ & $0.0717(8)$ & $0.0594(7)$ & $0.0065(6)$ & $-0.0069(5)$ & $0.0126(5)$ \\
\hline $\mathrm{Cl} 9$ & $0.0707(6)$ & $0.0483(7)$ & $0.0596(7)$ & $-0.0137(6)$ & $-0.0043(5)$ & $0.0006(5)$ \\
\hline $\mathrm{Cl} 10$ & $0.0670(6)$ & $0.0819(9)$ & $0.0672(8)$ & $0.0136(7)$ & 0.0194(5) & $-0.0176(6)$ \\
\hline Cl11 & $0.0535(5)$ & $0.0605(7)$ & $0.0574(7)$ & $0.0041(6)$ & $-0.0039(4)$ & $0.0120(5)$ \\
\hline Cl12 & $0.0847(7)$ & $0.0591(8)$ & $0.0614(8)$ & $-0.0239(6)$ & $0.0001(6)$ & $0.0011(6)$ \\
\hline 01 & $0.070(2)$ & $0.076(3)$ & $0.128(4)$ & $0.011(2)$ & $0.020(2)$ & $0.0118(19)$ \\
\hline $\mathrm{O} 2$ & $0.175(4)$ & $0.053(3)$ & $0.113(4)$ & $0.005(3)$ & $-0.013(3)$ & $-0.004(3)$ \\
\hline $\mathrm{O} 3$ & $0.070(2)$ & $0.108(4)$ & $0.162(5)$ & $-0.012(3)$ & $0.004(3)$ & $0.015(2)$ \\
\hline N1 & $0.080(3)$ & $0.041(3)$ & $0.062(3)$ & $-0.001(2)$ & $0.001(2)$ & $0.008(2)$ \\
\hline N2 & $0.063(2)$ & $0.079(3)$ & $0.074(3)$ & $0.005(3)$ & $0.012(2)$ & $0.006(2)$ \\
\hline N3 & $0.107(4)$ & $0.186(7)$ & $0.080(4)$ & $0.045(4)$ & $0.012(3)$ & $0.061(4)$ \\
\hline N4 & $0.165(6)$ & $0.072(4)$ & $0.125(5)$ & $-0.005(4)$ & $0.036(5)$ & $0.010(4)$ \\
\hline N5 & $0.193(7)$ & $0.105(6)$ & $0.084(4)$ & $0.004(4)$ & $0.024(4)$ & $0.076(5)$ \\
\hline N6 & $0.151(6)$ & $0.121(6)$ & $0.085(4)$ & $-0.007(4)$ & $-0.011(4)$ & $0.065(5)$ \\
\hline N7 & $0.142(7)$ & $0.247(13)$ & $0.117(7)$ & $-0.058(8)$ & $-0.056(6)$ & $0.130(8)$ \\
\hline N8 & $0.080(4)$ & $0.270(11)$ & $0.064(4)$ & $0.019(6)$ & $0.005(3)$ & $0.000(5)$ \\
\hline $\mathrm{C} 1$ & $0.097(4)$ & $0.054(3)$ & $0.063(3)$ & $-0.009(3)$ & $0.002(3)$ & $0.001(3)$ \\
\hline C2 & $0.065(3)$ & $0.080(4)$ & $0.073(4)$ & $0.015(3)$ & $-0.004(2)$ & $-0.010(3)$ \\
\hline C3 & $0.092(4)$ & $0.081(5)$ & $0.079(4)$ & $0.023(4)$ & $0.022(3)$ & $0.041(3)$ \\
\hline C4 & $0.130(5)$ & $0.052(4)$ & $0.079(4)$ & $-0.008(3)$ & $0.001(4)$ & $0.013(3)$ \\
\hline C5 & $0.088(4)$ & $0.067(4)$ & $0.083(4)$ & $-0.003(3)$ & $-0.008(3)$ & $-0.020(3)$ \\
\hline C6 & $0.066(3)$ & $0.035(3)$ & $0.059(3)$ & $0.005(2)$ & $0.001(2)$ & $-0.0001(2)$ \\
\hline C7 & $0.068(3)$ & $0.101(5)$ & $0.085(4)$ & $-0.018(4)$ & $0.008(3)$ & $-0.020(3)$ \\
\hline C8 & $0.090(4)$ & $0.108(6)$ & $0.080(5)$ & $0.008(4)$ & $-0.004(3)$ & $0.031(4)$ \\
\hline C9 & $0.142(6)$ & $0.057(4)$ & $0.088(5)$ & $0.010(4)$ & $0.028(4)$ & $0.015(4)$ \\
\hline C10 & $0.108(4)$ & $0.071(4)$ & $0.078(4)$ & $-0.006(4)$ & $-0.004(3)$ & $-0.028(3)$ \\
\hline C11 & $0.069(3)$ & $0.090(5)$ & $0.108(5)$ & $-0.003(4)$ & $-0.025(3)$ & $-0.014(3)$ \\
\hline C12 & $0.091(4)$ & $0.081(5)$ & $0.125(6)$ & $0.001(4)$ & $-0.021(4)$ & $0.033(4)$ \\
\hline C13 & $0.185(8)$ & $0.042(4)$ & $0.103(6)$ & $-0.016(4)$ & $0.031(5)$ & $-0.004(4)$ \\
\hline C14 & $0.085(4)$ & $0.093(5)$ & $0.054(3)$ & $-0.015(3)$ & $-0.010(3)$ & $-0.052(4)$ \\
\hline
\end{tabular}




\begin{tabular}{|c|c|c|c|c|c|c|}
\hline C15 & $0.048(3)$ & $0.166(7)$ & $0.065(4)$ & $0.026(5)$ & $-0.001(2)$ & $0.020(4)$ \\
\hline C16 & $0.098(4)$ & $0.070(4)$ & $0.082(4)$ & $-0.008(3)$ & $0.008(3)$ & $-0.027(3)$ \\
\hline C17 & $0.056(3)$ & $0.146(7)$ & $0.096(5)$ & $-0.001(5)$ & $0.006(3)$ & $0.015(4)$ \\
\hline C18 & $0.141(6)$ & $0.074(5)$ & $0.092(5)$ & $-0.002(4)$ & $0.026(4)$ & $0.039(4)$ \\
\hline C19 & $0.134(6)$ & $0.095(6)$ & $0.056(4)$ & $-0.021(4)$ & $0.019(4)$ & $-0.048(4)$ \\
\hline C20 & $0.060(3)$ & $0.097(5)$ & $0.066(4)$ & $-0.015(4)$ & $0.009(2)$ & $-0.006(3)$ \\
\hline $\mathrm{C} 21$ & $0.140(7)$ & $0.086(5)$ & $0.074(5)$ & $0.006(4)$ & $0.025(4)$ & $-0.027(5)$ \\
\hline $\mathrm{C} 22$ & $0.064(3)$ & $0.172(9)$ & $0.080(5)$ & $-0.015(5)$ & $0.012(3)$ & $0.016(5)$ \\
\hline C23 & $0.142(7)$ & $0.088(6)$ & $0.079(5)$ & $-0.003(4)$ & $-0.008(4)$ & $0.046(5)$ \\
\hline C24 & $0.143(7)$ & $0.101(6)$ & $0.074(5)$ & $-0.008(4)$ & $0.001(4)$ & $-0.039(5)$ \\
\hline C25 & $0.071(4)$ & $0.133(8)$ & $0.098(5)$ & $-0.022(6)$ & $-0.002(3)$ & $-0.004(5)$ \\
\hline C26 & $0.301(17)$ & $0.061(6)$ & $0.091(7)$ & $0.033(5)$ & $0.053(10)$ & $0.037(8)$ \\
\hline $\mathrm{C} 27$ & $0.118(7)$ & $0.138(10)$ & $0.191(13)$ & $-0.049(9)$ & $0.029(7)$ & $-0.075(7)$ \\
\hline C28 & $0.143(9)$ & $0.186(13)$ & $0.179(11)$ & $-0.016(11)$ & $-0.095(9)$ & $0.076(8)$ \\
\hline C29 & $0.232(13)$ & $0.068(6)$ & $0.146(10)$ & $0.030(6)$ & $0.040(10)$ & $0.001(8)$ \\
\hline C30 & $0.087(6)$ & $0.180(12)$ & $0.192(14)$ & $-0.076(10)$ & $0.046(7)$ & $-0.064(7)$ \\
\hline C31 & $0.172(10)$ & $0.102(8)$ & $0.162(10)$ & $0.069(7)$ & $0.093(8)$ & $0.089(7)$ \\
\hline C32 & $0.176(11)$ & $0.051(5)$ & $0.184(12)$ & $-0.044(7)$ & $0.082(8)$ & $-0.013(5)$ \\
\hline C33 & $0.090(5)$ & $0.214(12)$ & $0.092(6)$ & $-0.067(8)$ & $0.008(4)$ & $-0.036(6)$ \\
\hline C34 & $0.109(5)$ & $0.134(8)$ & $0.087(6)$ & $0.045(5)$ & $0.029(4)$ & $0.048(5)$ \\
\hline C35 & $0.116(6)$ & $0.056(4)$ & $0.132(7)$ & $-0.023(4)$ & $0.042(5)$ & $-0.010(4)$ \\
\hline
\end{tabular}

A projection of the structure on the (100) plane (Fig. 2) shows that each bismuth is surrounded by six $\mathrm{Cl}$ atoms, forming a distorsed octahedral configuration, with $\mathrm{Bi}-\mathrm{Cl}$ distances range from 2.6251(11) to 2.7990(12) A for the first octahedron and from $2.6852(10)$ to $2.7295(10) \AA$ for the second octahedron (see Table 4). These values are comparable with those reported by other reasearches [20]. In addition to the bond-lengths differences, the $\mathrm{Cl}-\mathrm{Bi}-\mathrm{Cl}$ angles within each $[\mathrm{BiCl} 6]^{3-}$ anion extend from 85.72 (4) to $94.52(4)^{\circ}$ and from $86.87(4)$ to $93.04(4)^{\circ}$ for cis and from $174.20(4)$ to $177.48(4)^{\circ}$ and $175.42(4)$ to $177.99(4)^{\circ}$ for trans arrangements. These values, which they listed in Table 4 , do not indicate any stereochemical activity of the $\mathrm{Bi}$ lone electron pair $[\mathbf{2 0 , 2 1 ]}$ but this distorsion is correlated to deformations resulting from strong hydrogen bond interactions [20,21], which involves all coordinated chlorine atoms [21]. The isolated nitrate anions play an important role in cohesion of the structure so they are located in the gaps left between the octahedra of bismuth and monoprotonated organic cations. In fact, the presence of those anions as a part of an anionic sublattice in the family of organic - inorganic hybrids was also found in other structure $[22,23]$. The seven organic template cations, $\left[\mathrm{C}_{5} \mathrm{H}_{5} \mathrm{NH}\right]^{+}$, reside within the crystal lattice and maintain the charge neutrality (see Fig. 1). Also, the C-N bond lentghs in organic cations are ranging 1.467 (12) - 1.504 (10) $\AA$ (see Table 4). The observed C-C distances in pyridinium are extended from 1.481 (16) to 1.533 (13) A (see Table 4). These values are comparable with those reported by other reaserchers [24]. Besides in this compound, the aromatic rings built up by $(\mathrm{C} 1, \mathrm{C} 2, \mathrm{C} 3, \mathrm{C} 4, \mathrm{C} 5, \mathrm{~N} 2),(\mathrm{C} 11, \mathrm{C} 12, \mathrm{C} 13, \mathrm{C} 14, \mathrm{C} 15, \mathrm{~N} 4)$ and $(\mathrm{C} 16, \mathrm{C} 17, \mathrm{C} 18, \mathrm{C} 19, \mathrm{C} 20, \mathrm{~N} 5)$ (rms deviation of fitted atoms equal to $0.0007 ; 0.0033$ and 0.0046 respectively) are more planar than those built pu by $(\mathrm{C} 6, \mathrm{C} 7, \mathrm{C} 8, \mathrm{C} 9, \mathrm{C} 10, \mathrm{~N} 3),(\mathrm{C} 21, \mathrm{C} 22, \mathrm{C} 23, \mathrm{C} 24, \mathrm{C} 25, \mathrm{~N} 6),(\mathrm{C} 26, \mathrm{C} 27, \mathrm{C} 28, \mathrm{C} 29, \mathrm{C} 30, \mathrm{~N} 7)$ and $(\mathrm{C} 31, \mathrm{C} 32, \mathrm{C} 33, \mathrm{C} 34, \mathrm{C} 35, \mathrm{~N} 8)$ ( $\mathrm{rms}$ deviation of fitted atoms equal to $0.0052 ; 0.0053 ; 0.0077$ and 0.0180 respectively). 


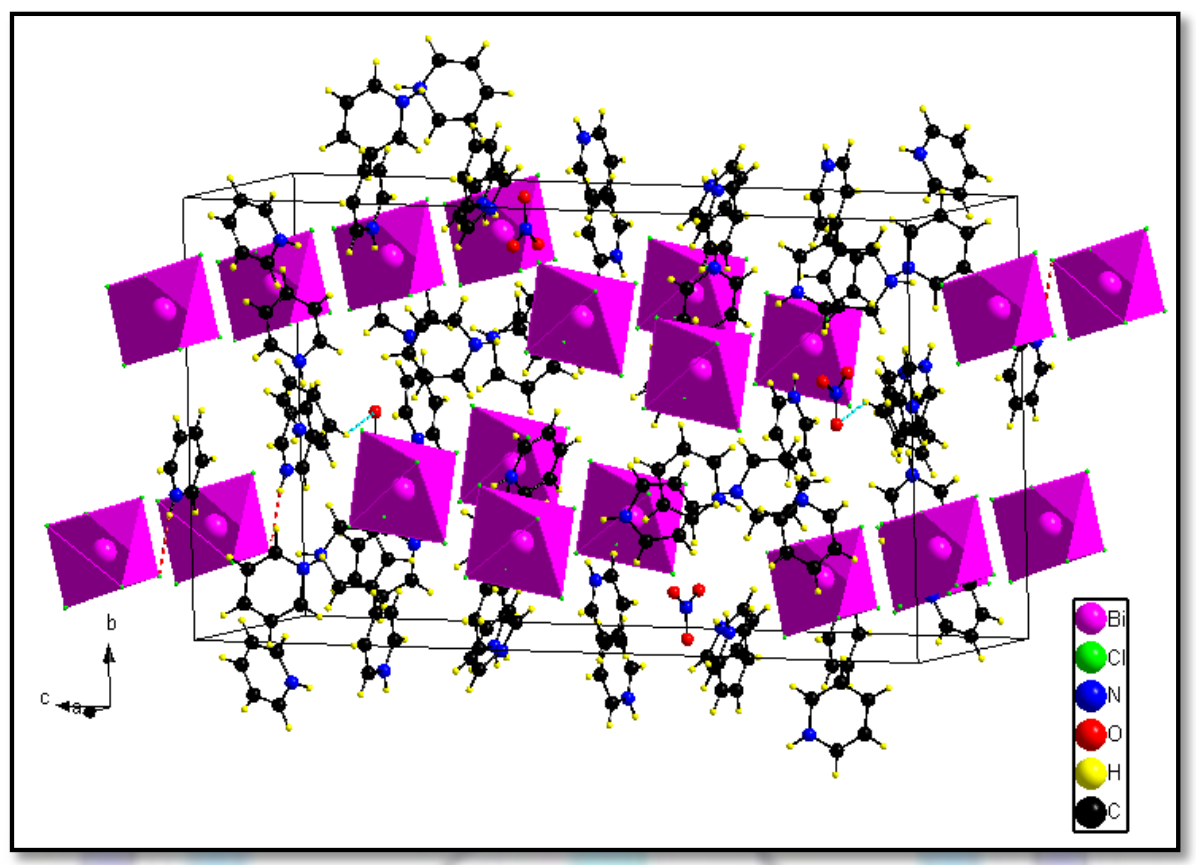

Figure 2 : Crystal packing of the $\left[\mathrm{C}_{5} \mathrm{H}_{5} \mathrm{NH}\right]_{7}\left(\mathrm{BiCl}_{6}\right)_{2}\left(\mathrm{NO}_{3}\right)$

Table 4: Selected bond $(\AA)$ lengths and angles $\left(^{\circ}\right)$ for $\left[\mathrm{C}_{5} \mathrm{H}_{5} \mathrm{NH}\right]_{7}\left(\mathrm{BiCl}_{6}\right)_{2}\left(\mathrm{NO}_{3}\right)$

\begin{tabular}{|c|c|c|c|}
\hline $\mathrm{Bi1-Cl1}$ & $2.7580(11)$ & $\mathrm{N} 1-\mathrm{O} 1$ & $1.255(5)$ \\
\hline $\mathrm{Bi1-Cl2}$ & $2.7390(10)$ & $\mathrm{N} 1-\mathrm{O} 2$ & $1.213(5)$ \\
\hline $\mathrm{Bi1-Cl3}$ & $2.7989(12)$ & $\mathrm{N} 1-\mathrm{O} 3$ & $1.184(5)$ \\
\hline $\mathrm{Bi1-Cl4}$ & $2.6597(11)$ & $\mathrm{N} 2-\mathrm{C} 1$ & $1.317(6)$ \\
\hline $\mathrm{Bi1-Cl5}$ & $2.6969(11)$ & C1-C2 & $1.373(7)$ \\
\hline $\mathrm{Bi1-Cl6}$ & $2.6251(11)$ & C2-C3 & $1.347(8)$ \\
\hline $\mathrm{Bi} 2-\mathrm{Cl} 7$ & $2.7179(10)$ & C3-C4 & $1.353(9)$ \\
\hline $\mathrm{Bi2-Cl8}$ & $2.7295(10)$ & N2-C5 & $1.307(7)$ \\
\hline Bi2-Cl9 & $2.7167(11)$ & C4-C5 & $1.318(8)$ \\
\hline $\mathrm{Bi} 2-\mathrm{Cl} 10$ & $2.6938(11)$ & N3-C6 & $1.330(8)$ \\
\hline $\mathrm{Bi} 2-\mathrm{Cl} 11$ & $2.6853(10)$ & C6-C7 & $1.261(7)$ \\
\hline \multirow[t]{12}{*}{$\mathrm{Bi} 2-\mathrm{Cl} 12$} & $2.6970(12)$ & C7-C8 & $1.336(9)$ \\
\hline & & C8-C9 & $1.324(9)$ \\
\hline & & N3-C10 & $1.403(9)$ \\
\hline & & C9-C10 & $1.314(9)$ \\
\hline & & C11 N4 & $1.334(8$ \\
\hline & & C11-C12 & $1.274(8)$ \\
\hline & & C12-C13 & $1.343(10)$ \\
\hline & & C13-C14 & $1.279(9)$ \\
\hline & & N4-C15 & $1.385(9)$ \\
\hline & & C14-C15 & $1.264(9)$ \\
\hline & & C16-N5 & $1.385(9)$ \\
\hline & & C16-C17 & $1.287(9)$ \\
\hline
\end{tabular}




\begin{tabular}{|c|c|c|c|}
\hline & & C17 -C18 & $1.362(9)$ \\
\hline & & C18-C19 & $1.341(9)$ \\
\hline & & C19-C20 & $1.264(9)$ \\
\hline & & C20-N5 & $1.308(8)$ \\
\hline & & N6-C21 & $1.374(10)$ \\
\hline & & C21-C22 & $1.310(10)$ \\
\hline & & C22-C23 & $1.342(10)$ \\
\hline & & C23-C24 & $1.320(10)$ \\
\hline & & N6-C25 & $1.261(9)$ \\
\hline & & C24-C25 & $1.278(10)$ \\
\hline & & N7-C26 & $1.320(14)$ \\
\hline & & C26-C27 & $1.283(14)$ \\
\hline & & C27-C28 & $1.256(10)$ \\
\hline & & C28-C29 & $1.283(12)$ \\
\hline & & N7-C30 & $1.283(14)$ \\
\hline & & C29-C30 & $1.230(12)$ \\
\hline & & N8-C31 & $1.416(13)$ \\
\hline & & C31-C32 & $1.233(13)$ \\
\hline & & C32-C33 & $1.260(13)$ \\
\hline & & C33-C34 & $1.246(11)$ \\
\hline & & N8-C35 & $1.343(10)$ \\
\hline & & C34-C35 & $1.297(11)$ \\
\hline Cl6-Bi1-Cl4 & $91.94(4)$ & O3-N1-O2 & $121.6(5)$ \\
\hline Cl6-Bi1-Cl5 & $91.28(4)$ & O3-N1-O1 & 119.8(5) \\
\hline Cl4-Bi1-Cl5 & $89.66(4)$ & O2-N1-O1 & $118.6(5)$ \\
\hline Cl6-Bi1-Cl2 & $88.50(3)$ & C5-N2-C1 & $121.8(5)$ \\
\hline $\mathrm{Cl} 4-\mathrm{Bi1}-\mathrm{Cl} 2$ & $87.02(3)$ & C7-C6-N3 & $121.7(5)$ \\
\hline $\mathrm{Cl} 5-\mathrm{Bi} 1-\mathrm{Cl} 2$ & $176.67(4)$ & $\mathrm{N} 2-\mathrm{C} 1-\mathrm{C} 2$ & $118.8(5)$ \\
\hline Cl6-Bi1-Cl1 & $90.56(4)$ & C3-C2-C1 & 119.2(5) \\
\hline Cl4-Bi1-Cl1 & $177.48(4)$ & C12-C11-N4 & $120.8(6)$ \\
\hline Cl5-Bi1-Cl1 & $89.98(4)$ & C15-C14-C13 & 122.1(5) \\
\hline $\mathrm{Cl} 2-\mathrm{Bi} 1-\mathrm{Cl}$ & $93.34(3)$ & C6-N3-C10 & 117.7(5) \\
\hline Cl6-Bi1-Cl3 & $174.19(4)$ & C9-C8-C7 & 119.1(6) \\
\hline Cl4-Bi1-Cl3 & $88.35(4)$ & C2-C3-C4 & $119.2(5)$ \\
\hline Cl5-Bi1-Cl3 & $94.52(4)$ & C9-C10-N3 & $118.6(6)$ \\
\hline $\mathrm{Cl} 2-\mathrm{Bi} 1-\mathrm{Cl} 3$ & $85.72(4)$ & C6-C7-C8 & $122.0(6)$ \\
\hline $\mathrm{Cl} 1-\mathrm{Bi} 1-\mathrm{Cl} 3$ & $89.19(4)$ & C10-C9-C8 & $120.9(6)$ \\
\hline Cl11-Bi2-Cl10 & $91.48(4)$ & N2-C5-C4 & $120.8(5)$ \\
\hline Cl11-Bi2Cl12 & $90.72(4)$ & C5-C4-C3 & $120.1(6)$ \\
\hline Cl10-Bi2-Cl12 & $87.23(4)$ & C11-N4-C15 & $117.4(6)$ \\
\hline Cl11-Bi2-Cl9 & $89.56(4)$ & C14-C15-N4 & 119.4(5) \\
\hline
\end{tabular}




\begin{tabular}{|c|c|c|c|}
\hline Cl10-Bi2-Cl9 & $90.77(4)$ & C11-C12-C13 & $119.9(6)$ \\
\hline Cl12-Bi2-Cl9 & 177.99(4) & C14-C13-C12 & $120.2(6)$ \\
\hline Cl11-Bi2-Cl7 & $88.65(3)$ & C19-C20-N5 & $120.3(6)$ \\
\hline $\mathrm{Cl} 10-\mathrm{Bi2}-\mathrm{Cl} 7$ & $\begin{array}{l}177.21(4) \\
\end{array}$ & C17-C16-N5 & $119.0(6)$ \\
\hline $\mathrm{Cl} 12-\mathrm{Bi} 2-\mathrm{Cl} 7$ & 89.98(4) & C19-C18-C17 & $118.6(6)$ \\
\hline Cl9-Bi2-Cl7 & $92.02(4)$ & C20-C19-C18 & $122.0(6)$ \\
\hline Cl11-Bi2-Cl8 & $175.42(4)$ & C16-C17-C18 & 119.9(6) \\
\hline $\mathrm{Cl} 10-\mathrm{Bi2}-\mathrm{Cl} 8$ & $93.05(4)$ & C20-N5-C16 & $120.3(5)$ \\
\hline Cl12-Bi2-Cl8 & $90.23(4)$ & C25-N6-C21 & $121.1(7)$ \\
\hline Cl9-Bi2-Cl8 & $89.65(4)$ & C35-N8-C31 & $114.9(7)$ \\
\hline Cl7-Bi2-Cl8 & $86.87(4)$ & C21-C22-C23 & $119.3(6)$ \\
\hline & & N6-C25-C24 & $121.2(7)$ \\
\hline & & C33-C34-C35 & $120.5(8)$ \\
\hline & & C34-C35-N8 & $119.9(7)$ \\
\hline & & C32-C31-N8 & $119.5(8)$ \\
\hline & & C24-C23-C22 & $119.6(7)$ \\
\hline & & C25-C24-C23 & $120.9(7)$ \\
\hline & & C31-C32-C33 & $121.8(10)$ \\
\hline & & C22-C21-N6 & $117.9(7)$ \\
\hline & & C34-C33-C32 & 123.1(9) \\
\hline & & C30-N7-C26 & $119.0(8)$ \\
\hline & & C27-C26-N7 & 118.3(9) \\
\hline & & C28-C27-C26 & 118.3(9) \\
\hline & & C27-C28-C29 & $124.8(9)$ \\
\hline & & C29-C30-N7 & 123.1(9) \\
\hline & & C30-C29-C28 & 116.5(9) \\
\hline
\end{tabular}

In the same time, the hydrogen atoms bonded to nitrogen atoms participate in the formation of $\mathrm{N}-\mathrm{H}$... $\mathrm{Cl}$ hydrogen bonding with chlorine atoms of $\mathrm{BiCl}_{6}{ }^{3-}$ anions and in the formation of $\mathrm{N}-\mathrm{H}$...O hydrogen bonding with to oxygen atoms of isolaten nitrate anions (Fig. 3). All of these hydrogen bonds, $\mathrm{N}-\mathrm{H}^{\cdots} \mathrm{Cl}$ and $\mathrm{N}-\mathrm{H}$... (see Table 5) give rise to a three - dimensional network in the structure and add stability of this compound. 


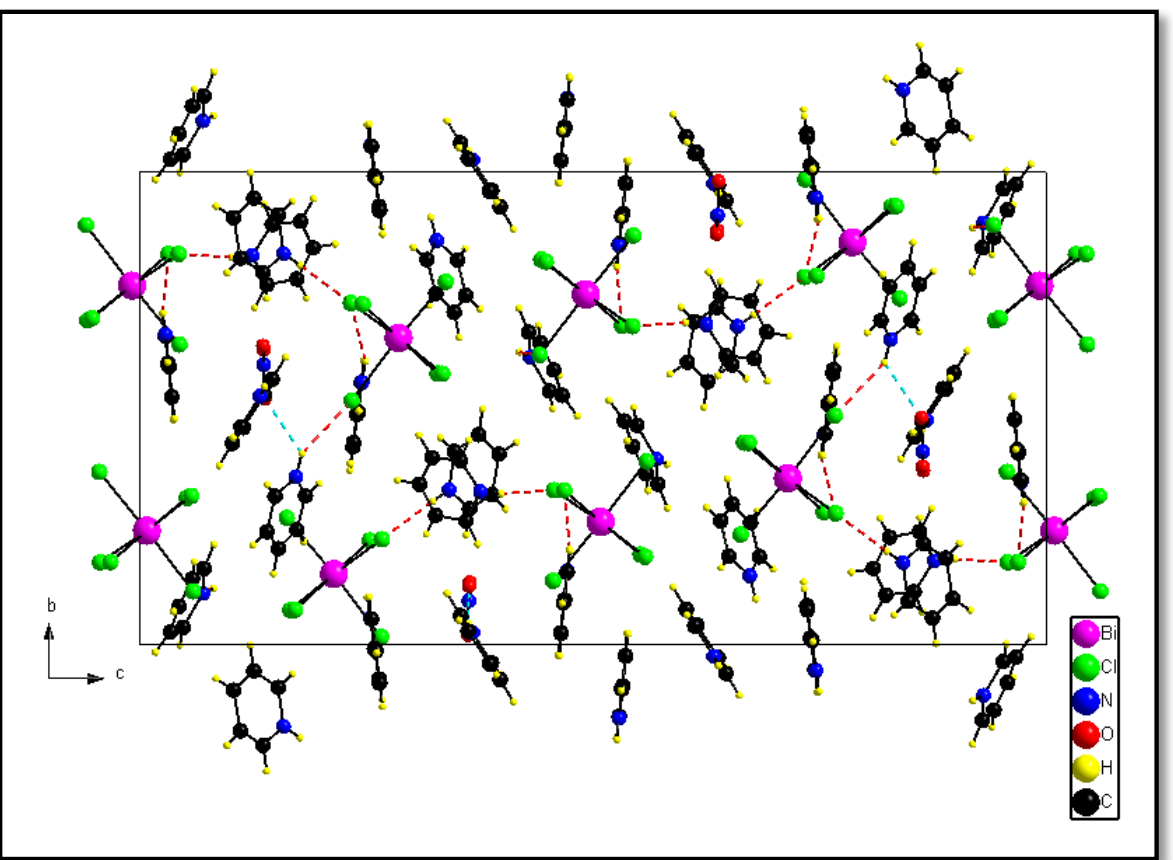

Figure 3: Hydrogen bond between the constituents of the structure of $\left[\mathrm{C}_{5} \mathrm{H}_{5} \mathrm{NH}\right]_{7}\left(\mathrm{BiCl}_{6}\right)_{2}\left(\mathrm{NO}_{3}\right)$ along the axis a

Table 5: Hydrogen bonds for $\left[\mathrm{C}_{5} \mathrm{H}_{5} \mathrm{NH}_{7}\left(\mathrm{BiCl}_{6}\right)_{2}\left(\mathrm{NO}_{3}\right)\right.$

\begin{tabular}{|c|c|c|c|c|}
\hline $\mathbf{D}-\mathbf{H} \cdots \mathbf{A}$ & $\mathbf{d}(\mathbf{D}-\mathbf{H})$ & $\mathbf{d}(\mathbf{H} \cdots \mathbf{A})$ & $\mathbf{d}(\mathbf{D} \cdots \mathbf{A})$ & $<$ (DHA) \\
\hline $\mathrm{N} 2-\mathrm{H} 2 \cdots \mathrm{O} 1$ & 0,860 & 1,946 & 2,760 & 157,44 \\
\hline $\mathrm{N} 2-\mathrm{H} 2 \cdots \mathrm{O} 2$ & 0,860 & 2,658 & 3,401 & 145,41 \\
\hline $\mathrm{N} 2-\mathrm{H} 2 \cdots \mathrm{N} 1$ & 0,860 & 2,676 & 3,518 & 177,13 \\
\hline $\mathrm{N} 3-\mathrm{H} 3 \cdots \mathrm{Cl} 1$ & 0,860 & 2,867 & 3,726 & 133,22 \\
\hline $\mathrm{N} 4-\mathrm{H} 4 \mathrm{~A} \cdots \mathrm{O} 2$ & 0,860 & 2,496 & 3,597 & 134,40 \\
\hline $\mathrm{N} 4-\mathrm{H} 4 \mathrm{~A} \cdots \mathrm{Cl} 3$ & 0,860 & 2,942 & 3,623 & 156,55 \\
\hline $\mathrm{N} 5-\mathrm{H} 5 \mathrm{~A} \cdots \mathrm{Cl} 1$ & 0,860 & 2,818 & 3,466 & 169,85 \\
\hline $\mathrm{N} 6-\mathrm{H} 6 \mathrm{~A} \cdots \mathrm{Cl}{ }^{\prime}$ & 0,860 & 2,616 & 3,540 & 129,15 \\
\hline $\mathrm{N} 7-\mathrm{H} 7 \mathrm{~A} \cdots \mathrm{Cl9}$ & 0,860 & 2,934 & 3,508 & 167,45 \\
\hline $\mathrm{N} 8-\mathrm{H} 8 \mathrm{~A} \cdots \mathrm{Cl} 10$ & 0,860 & 2,664 & & \\
\hline
\end{tabular}

Codes de symétries: (i) $x-1 / 2,-y+3 / 2, z-1 / 2$

\section{Conclusion}

In this study, a novel organic-inorganic bismuth-chloride based compound with general formula $\left[\mathrm{C}_{5} \mathrm{H}_{6} \mathrm{NH}_{7}\left(\mathrm{BiCl}_{6}\right)_{2}\left(\mathrm{NO}_{3}\right)\right.$ has been synthesized. Single-crystal X-ray diffraction showed the formation of title compound consisting of two independent octahedra of Bismuth, seven monoprotonated organic cations and isolated nitrate anion. Those components are connected themselves by two types of intermolecular hydrogen bonds.

\section{ACKNOWLEDGEMENTS}

This work is supported by the Tunisian National Ministry of Higher Education and Scientific Research and the Crystallography Group which it is a part of the Laboratory of Crystallography and Materials Science (CRISMAT) Joint Research Unit 6508. 


\section{REFERENCES}

[1] L. Sobczyk, R. Jakubas, J. Zaleski, Pol. Chem. 71 (3) (1997) 265.

[2] H. Ishihara, K. Yamada, T. Okuda, A. Weiss, Bull. Chem. Soc. Jpn. 66 (2) (1993) 380.

[3] A. Kallel, J. W. Bats, Acta Crystallogr. , Sect.C 41 (7) (1985) 1022-1024.

[4] M. Bujak, R. J. Angel, J. Solid State Chem. 180 (11) (2007) 3026.

[5] M. Bujak, R. J. Angel, J. Phys . Chem.B.110 (2006) 10322.

[6] G. Xu, Y. Li, W. Zhou, G. Wang, L. Cai, M. Wang, G. Guo, J. Huang, G. Bator, R. Jakubas, J. Mater. Chem. 19(15) (2009)2179.

[7] R. Jakubas, U. Krzewska, G. Bator, L. Sobczyk, Ferroelectrics 77(1) (1988)129.

[8] R. Jakubas, Solid State Commun. 60 (4) (1986)389.

[9] J. Zaleski, Cz. Pawlaczyk, R. Jakubas, H. -G. Unruh, J. Phys. : Condens. Mater 12 (33) (2000) 7509-7521.

[10] A. Miniewicz, J. Sworakowski, R. Jakubas, M. Bertault, C. Ecolivet, Ferroelectrics 94 (1) (1989) 323.

[11] M. Hall, M. Nunn, M. Beglev, D. Bryan Soverby, J. Chem. Soc. , Dalton Trans. 6 (1986) 1231.

[12] R. Jakubas, Z. Ciunik, G. Bator, Phys.Rev B 67 (2) (2003) 241031 - 241036 . art. N 024103.

[13] A. Piecha, A. Pietraszko, G. Bator, R. Jakubas, J. Solid State Chem. 181 (5) (2008) 1155.

[14] R. Jakubas, J. Jozkow, G. Bator, J. Korean Phys. Soc. 32 (SUPLL 1) (1998) S302.

[15] J. Jozkow, W. Medycki, J. Zaleski, R. Jakubas, G. Bator, Z. Ciunik, Phys. Chem. Chem. Phys. 3 (15) (2001) 3222.

[16] J. Jozkow, R. Jakubas, G. Bator, A. Pietraszko, J. Chem. Phys. 114 (16) (2001) 7239.

[17] G. Bator, R. Jakubas, J. Baran, Vib . Spectrosc. 45 (1) (2007) 36.

[18] G. M. Sheldrick, SHELXS-97, Program for the refinement of crystal structures, 1990.

[19] G. M. Sheldrick, SHELXL-97, Program for the refinement of crystal structures, 1997.

[20] S. Nasri, F. Zouari and H. El Féki, J. Chem. Crystallogr. 38 (2008) 729-732.

[21] I. Baccar, F. Zouari, N. Chniba-Boudjada and P. Bordet, Jpn. Soc. Anal. Chem. 26 (2010) 15-16.

[22] C. Hrizi, C. Chaker and S. Chaabouni, lonics 17 (2011) 545-553.

[23] Y. Hua Gao, X. Jia Liu, L. Sun and W.-J. Le, Acta Crystallogr. E 67 (2011) 1688.

[24] G. M. Golzar Hossain, Acta Cryst. E69, (2013) 402. 